http://jmscr.igmpublication.org/home/ ISSN (e)-2347-176x ISSN (p) 2455-0450 crossref DOI: https://dx.doi.org/10.18535/jmscr/v9i1.37

\title{
Clinical Profile and Outcomes of Patients Admitted with COVID-19 Infection-Report from a Tertiary Hospital
}

\author{
Authors \\ Harharpreet Kaur ${ }^{1 *}$, Manpreet Singh ${ }^{2}$, Kiranjit Kaur $^{3}$, Arvinder Kaur ${ }^{4}$, \\ Randhir Singh $^{5}$, Nehal Patel ${ }^{6}$ \\ ${ }^{1}$ Professor and Head, Medicine Department, MM Medical College, Kumarhatti, Solan \\ ${ }^{2}$ Professor, ENT, Department and Medical Superintendent, MM Medical College, Solan \\ ${ }^{3}$ Professor \& Head, Biochemistry Deptt, MM Medical College, Kumarhatti, Solan \\ ${ }^{4}$ Senior Resident, Department of Medicine, MM Medical College, Kumarhatti, Solan \\ ${ }^{5,6}$ Junior Resident, Department of Medicine, MM Medical College, Kumarhatti, Solan \\ *Corresponding Author \\ Dr Harharpreet Kaur \\ Professor Medicine, MM Medical College, Kumarhatti, Distt. Solan, Himachal Pradesh, India
}

\begin{abstract}
Introduction: Coronavirus disease (COVID-19) is caused by severe acute respiratory syndrome coronavirus 2 (SARS-CoV-2). Symptoms may be mild which do not need hospital admission. However some patients progress to acute respiratory failure, Adult Respiratory Syndrome (ARDS)/sepsis and require intensive care. There is paucity of data regarding the clinical course of the disease.

Aims and Objectives: To study the clinicodemographic characteristics, associated comorbid conditions and outcomes of COVID 19 patients admitted in a tertiary hospital.

Methods: 185 consecutive patients with confirmed diagnosis of COVID-19 who were admitted from September 2020 to 31st December 2020 in a tertiary hospital were studied. Only patients with moderate and severe disease were admitted. Rests were advised home quarantine. They were given medical treatment as per Indian guidelines and besides the patient profile their clinical course, oxygen requirement and need for ventilator support and outcomes were studied.

Results: Age ranged from 30-86 years. 62\% of patients were between the age of 51-70 years.68.1\% patients were males and $31.9 \%$ were females.91.4\%patients were admitted with shortness of breath (SOB).Other complaints included fever (10.3\%) and cough(18.4\%) .68\% of the subjects had associated comorbid conditions. Diabetics constituted $49.2 \%$ and hypertensives $41.1 \%$ of these cases.59\% of patients were stabilised on low flow oxygen/without oxygen, $8 \%$ required high flow oxygen, $29 \%$ needed non invasive ventilation and $3 \%$ had to be mechanically ventilated. $57 \%$ of patients who required noninvasive ventilation were diabetics. Significantly higher comorbidity was present among patients with severe disease as compared to those with moderately severe disease. (Pearson chi2 $(1)=22.9781 \mathrm{Pr}=0.000) .83 \%$ of all admitted patients recoveredcompletely. $70 \%$ cases who received non invasive ventilation recovered and had no residual complication.5\% were referred or left the hospital. The hospital stay ranged from 1 to 36 days. Mortality was 12\%.3 patients who expired reported very late and were referred from other centres.

Conclusion: The patients who were admitted with COVID-19 were elderly with a male predominance. Comorbiditywas significantly associated with severe disease. Recovery rate is good with early and aggressive ICU care.

Keywords: COVID-19, Patient profile; Comorbidities, Severe Disease; Outcomes.
\end{abstract}




\section{Introduction}

Patients with SARS-CoV-2 infection may be asymptomatic but can also develop serious complications requiring hospitalization and admission to intensive care unit (ICU). It is believed that the clinical course is affected by underlying health conditions or comorbidities. Our study aimed to examine the demographic characteristics, clinical profile, associated comorbid conditions, the disease progression, clinical course and most importantly the outcomes of such patients who were hospitalized in our institution.

\section{Methods}

This study was a retrospective study, and 185 consecutive patients with confirmed diagnosis of COVID-19 who tested positive on real-time reverse transcriptase polymerase chain reaction (RT-PCR) assay for SARS-CoV-2 on a throat and/or a nasopharyngeal swab who were admitted to MM Medical College \& hospital, Solan, Himachal Pradesh, India from September 2020 to 31st December 2020 were enrolled. Besides these patients those with high CT score and antenatal mothers with any medical complaints were also studied.

COVID -19 patients were classified into mild, moderate, severe and critical as per Govt of India COVID-19 treatment guidelines. Mild with no evidence of hypoxemia with Sp02 above 94\% were advised home quarantine. Only moderate, severe and critical patients having evidence of hypoxemia or shortness of breath or high grade fever were admitted to the hospital.

- Moderate -The patients were classified as moderate if $\mathrm{Sp02}$ was $94 \%-90 \%$ in room air and the patient had no signs of severe disease and was stable with low flow oxygen.

- Severe patients were those with $\mathrm{SpO} 2$ : < 90\% room air RR: >30/min.

- Critical-were those with ARDS and sepsis They were given standard treatment as per Indian guidelines. Our admitted patients were managed according to the following protocol in our institution. They could be divided in general into 4 categories and 2 groups.

(Group 1)

1. Those who were by and large stable with low flow oxygen (upto 10 litres per minute via nasal prongs or mask)) or even without oxygen and could be categorized into the moderately severe group. (Group 1)

Group 11

2. Those requiring high flow oxygen (more than 10 litres per minute requiring mask with reservoir bag)

3. Those requiring non invasive ventilation (NIV)

4. Those requiring mechanical ventilation The category 2,3 and 4 were clubbed into Severe/critical group or the Group 11.The particulars of the patients were noted. Chi-square test $\left(\chi^{2}\right)$ was used for checking statistical significance of the association using Strata software.

\section{Results}

The age group of the patients is given in Table 1 Most of the patients were between the ages of 5170 years. Thus the older age individuals are at greater risk of requiring hospitalisation.

Sex 126(68.1\%) patients were males and $59(31.9 \%)$ were females showing that Covid19 infection affects the males more often.

Symptoms-169 (91.4\%)patients were admitted with shortness of breath (SOB). Other symptoms were cough in 34 patients $(18.4 \%)$, sore throat in $21(11.4 \%)$, fever in $19(10.3 \%)$ Thus majority of patients in our study were admitted with SOB.

We admitted only patients who had high grade fever, low oxygen saturation or shortness of breath. Those with mild symptoms like cough etc were not admitted therefore the percentage of patients with these symptoms is comparatively less in our study as compared to those with SOB.

Respiratory support The patients requiring respiratory support are given in Table 2. 
Comorbid Conditions Associated comorbid conditions are enlisted in Table 3 which shows that majority of the admitted patients had some or the other comorbidity.30 patients (57\%) of patients who required noninvasive ventilation were diabetics. There was significant correlation between comorbidity and patients with severe disease (Group 2) as compared to moderately severe disease (Group 1) (Pearson chi2 (1) $=22.9781 \mathrm{Pr}=0.000)$. (Table 4$)$

Outcomes- are depicted in Fig 1.154 pts (83\%)of all admitted patients recovered completely. 5\% were referred or left the hospital.38/54 patient (70\%) cases who received non invasive ventilation recovered and had no residual complication. 4 patients were discharged while on oxygen.1 patient needed intermittent oxygen 1 month after discharge. Rest had recovered completely though some complained of general weakness.

Mortality was $12 \%$ (22 patients). 3 patients who expired reported very late and were referred from other centres. Out of those who were mechanically ventilated patient there was no survivor who included a pregnant lady out of 38 antenatal women (not included in medical patients) who were aymptomatic and underwent normal (24) or caesariansection (14) and were discharged without complication.

Hospital stay ranged from 1 to 36 days. The hospital stay is shown in fig 2

4 patients not included in the list were Rt-PCR negative but high CT severity score on CT scan but recovered after supportive treatment and oxygen without need for ventilation.

Table 1 Age Distribution

\begin{tabular}{|l|c|c|}
\hline Age group & No. of cases & Percentage \\
\hline 40 & 19 & $10.3 \%$ \\
\hline $41-50$ & 32 & $17.3 \%$ \\
\hline $51-60$ & 61 & $33.0 \%$ \\
\hline $61-70$ & 53 & $28.6 \%$ \\
\hline 70 & 20 & $10.8 \%$ \\
\hline Total & 185 & $100.0 \%$ \\
\hline
\end{tabular}

Table 2 Respiratory Support

\begin{tabular}{|l|c|c|}
\hline & No. of cases & Percentage \\
\hline Patients stable on low flow oxygen/without oxygen (Group 1) & 110 & $59 \%$ \\
\hline Patients requiring high flow oxygen & 15 & $8 \%$ \\
\hline Patients requiring non invasive ventilation & 54 & $29 \%$ \\
\hline Patients requiring mechanical ventilation & 6 & $3 \%$ \\
\hline
\end{tabular}

Table 3

\begin{tabular}{|l|c|c|}
\hline COMORBIDITY & No. of cases & Percentage \\
\hline Overall Comorbidity & 126 & $68 \%$ \\
\hline Diabetes Mellitus & 91 & $49.2 \%$ \\
\hline Hypertension & 76 & $41.1 \%$ \\
\hline Chronic obstructive airway disease & 13 & $7.0 \%$ \\
\hline Obesity & 20 & $11 \%$ \\
\hline Alcoholism & 6 & $3.0 \%$ \\
\hline Anemia & 1 & $0.5 \%$ \\
\hline Appendicular lump & 1 & $0.5 \%$ \\
\hline Atrial Fibrillation & 1 & $0.5 \%$ \\
\hline CA Breast/Ca prostate & 2 & $1 \%$ \\
\hline Coronary artery disease/chest pain & 3 & $2 \%$ \\
\hline Chronic kidney disease & 2 & $1.0 \%$ \\
\hline Epistyxsis & 1 & $0.5 \%$ \\
\hline Hypothyroidism & 6 & $3.2 \%$ \\
\hline Severe infection & 3 & $2.0 \%$ \\
\hline Pregnancy & 1 & $0.5 \%$ \\
\hline
\end{tabular}




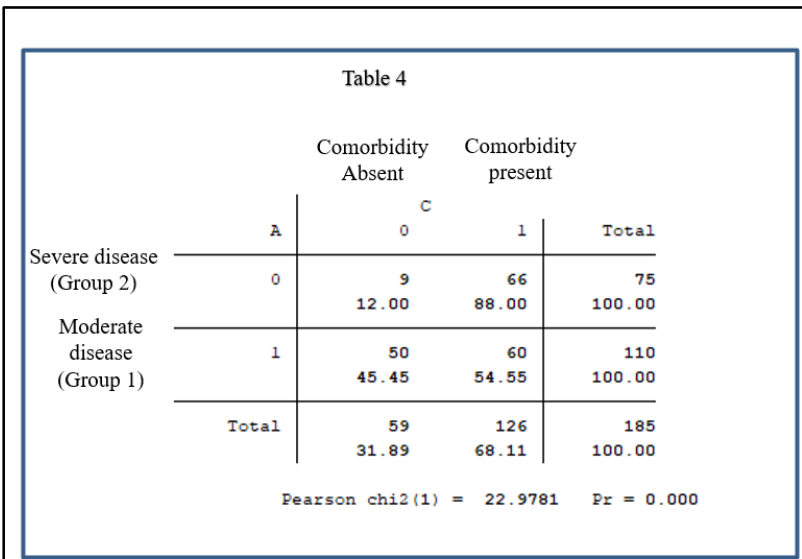

Showing Correlation between Comorbidity and Severity of Disease

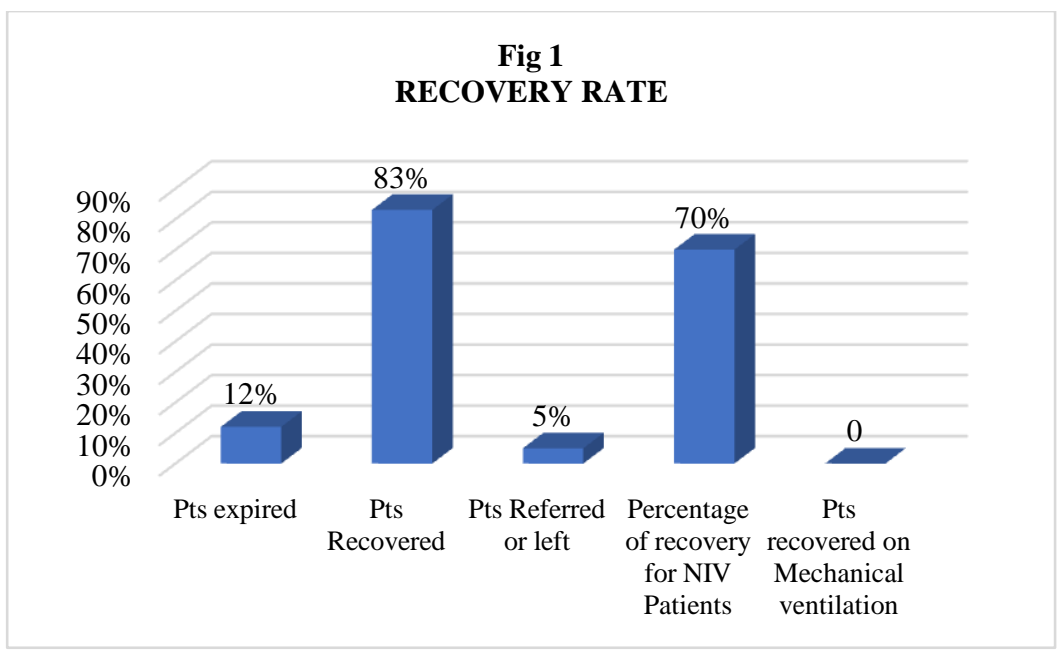

Fig 2

HOSPITAL STAY

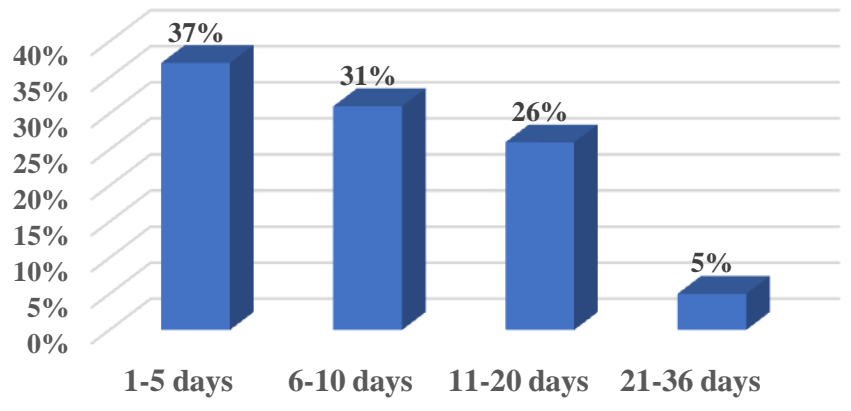

\section{Discussion}

In our study more of older individuals were hospitalised in conformity with other reports according to which the greatest risk for severe illness from COVID-19 is among those aged 85 or older. ${ }^{1,2}$ Male sex was preferentially targeted by the COVID -19 infection also shown by some of other Indian studies. ${ }^{3,4}$
$126(68 \%)$ of our patients who were hospitalized had some or the other comorbidity.(Table 3) Keeping in mind the prevalence of diabetes as around $11.9 \%$ in India ,91 (49.2\%) of our admitted cases had diabetes. 34 out of 54 (57\%) patients who required noninvasive ventilation (and thus severe disease) were diabetics. Thus diabetics needed hospitalization and also ICU care more 
frequently. Similarly hypertension was an important comorbidity present in $42 \%$ of our patients. (Table 3)Two of these patients had only mild hypertension.2 patients had associated malignancy.6 also gave history of hypothyroidism and 13 of COPD. (Table 3)

In a meta-analysis study on COVID-19 comorbidities by Paudel et $\mathrm{al}^{5}$ of a total of 1786 patients the most common comorbidities identified in these patients were hypertension $(15.8 \%)$, cardiovascular and cerebrovascular conditions $(11.7 \%)$, and diabetes $(9.4 \%)$. The less common comorbidities were malignancy $(1.5 \%)$, respiratory illnesses $(1.4 \%)$ and renal disorders $(0.8 \%)$.Our group had higher percentage of diabetes and hypertension as they were all admitted patients with moderate to severe disease. One pregnant patient developed severe ARDS and was intubated . She underwent caesarian section but could not be saved. She had additionally severe septicemia with a TLC of 22000 . It is to be noted that 38pregnant patients were admitted during this period for delivery. They were not included in the list of medical patients. All were asymptomatic and underwent caesarian section ${ }^{(14)}$ and the rest had normal delivery and were discharged without recording any complication.

$12 \%$ of all patients expired.The overall mortality reported by different studies was $10.5 \%$, (Indian study) ${ }^{3}$ USA $(21.1 \%)^{6}$ and UK $(26 \%)^{7}$.

$29 \%$ of severe group (ICU) expired. Quah et $\mathrm{al}^{8}$ reported a mortality of $25.7 \%$ among ICU patients.

$83 \%$ of all admitted patients recovered which included patients with moderate and severe disease. 38 out of 54 patients (70\%) who received non invasive ventilation recovered. $5 \%$ were referred or left.1patientout of the NIV group was on intermittent oxygen one month after discharge. Rest were doing well with no residual symptoms. Some complained of weakness though. No patient in the group who had to be mechanically ventilated recovered. It is to be noted that most of these patients reported very late.

\section{Conclusion}

Elderly individuals especially of male sex and comorbidities such as diabetes and hypertension were risk factors for hospitalisation and progression to severe disease. Early aggressive ICU care particularly non invasive ventilation plays an important role in management of COVID-19 patients with severe disease along with drug therapy. Knowledge of the factors affecting the outcome and disease progression can serve as guideline for more efficient management of COVID-19 patients in future.

\section{References}

1. CDC. Coronavirus (COVID-19): symptoms of coronavirus. Centers for Disease Control and Prevention. 2020.

2. Liu K, Chen Y, Lin R, Han K. Clinical features of COVID-19 in elderly patients: a comparison with young and middle-aged patients. J Inf Secur. 2020;15(30)

3. Budhiraja S,Soni Aet al, Clinical Profile of First 1000 COVID-19 Cases Admitted at Tertiary Care Hospitals and the Correlates of their Mortality: An Indian Experience. medRxiv preprint doi: https://doi.org/10.1101/2020.11.16.202322 23; November 18, 2020.

4. Mohan A, Tiwari P, Bhatnagar S, et al Clinico-demographic profile \& hospital outcomes of COVID-19 patients admitted at a tertiary care centre in north India. Indian J Med Res. 2020 Jul \& Aug;152(1 \& 2):61-69.

5. Paudel SS. A meta-analysis of 2019 novel coronavirus patient clinical characteristics and comorbidities. Research Square. 2020. 10.21203/rs.3.rs-21831/v1

6. Argenziano MG, Bruce SL, Slater CL, et al. Characterization and clinical course of 1000 patients with coronavirus disease 2019 in New York: Retrospective case series.BMJ 2020;369:m1996.

7. Docherty AB, Harrison EM, Green CA. Features of 20133 UK patients in 
hospitalwith covid-19 using the ISARIC WHO Clinical Characterisation Protocol: Prospective observational cohort study. BMJ 2020;369:m1985

8. Quah et al. Mortality rates of patients with COVID-19 in the intensive care unit: a systematic review of the emerging literature.Critical Care (2020) 4:285 https://doi.org/10.1186/s13054-02003006-1. 